\title{
LOWER BOUNDS IN THE MATRIX CORONA THEOREM AND THE CODIMENSION ONE CONJECTURE.
}

\author{
SERGEI TREIL
}

\begin{abstract}
Main result of this paper is the following theorem: given $\delta$, $0<\delta<1 / 3$ and $n \in \mathbb{N}$ there exists an $(n+1) \times n$ inner matrix function $F \in H_{(n+1) \times n}^{\infty}$ such that

$$
I \geq F^{*}(z) F(z) \geq \delta^{2} I \quad \forall z \in \mathbb{D},
$$

but the norm of any left inverse for $F$ is at least $[\delta /(1-\delta)]^{-n} \geq$ $\left(\frac{3}{2} \delta\right)^{-n}$. This gives a lower bound for the solution of the Matrix Corona Problem, which is pretty close to the best known upper bound $C$. $\delta^{-n-1} \log \delta^{-2 n}$ obtained recently by T. Trent [16]. In particular, both estimates grow exponentially in $n$; the (only) previously known lower bound $C \delta^{-2} \log \left(\delta^{2} n+1\right)$ (obtained by the author [13]) grew logarithmically in $n$. Also, the lower bound is obtained for $(n+1) \times n$ matrices, thus giving the negative answer to the so-called "codimension one conjecture." Another important result is Theorem 2.4 connecting left invertiblity in $H^{\infty}$ and co-analytic orthogonal complements.
\end{abstract}

\section{Contents}

Notation

$0 . \quad$ Introduction 2

1. Main results 4

2. Co-analytic complements and their role in the Corona Problem 5

3. Main construction and the proof of Theorem 1.1. 10

4. Proof of Theorem 1.2. 12

5. Some open problems. 14

$\begin{array}{ll}\text { References } & 14\end{array}$

\section{Notation}

$\mathbb{D} \quad$ Open unit disk in the complex plane $\mathbb{C}, \mathbb{D}:=\{z \in \mathbb{C}:|z|<1\}$;

$\mathbb{T} \quad$ Unit circle, $\mathbb{T}:=\partial \mathbb{D}=\{z \in \mathbb{C}:|z|=1\} ;$

Date: March, 2003.

S. Treil is partially supported by the NSF grant DMS-0200584. 
$H^{2}, H^{\infty} \quad$ Hardy classes of analytic functions,

$$
H^{p}:=\left\{f \in L^{p}(\mathbb{T}): \hat{f}(k):=\int_{\mathbb{T}} f(z) z^{-k} \frac{|d z|}{2 \pi}=0 \text { for } k<0\right\} .
$$

Hardy classes can be identified with spaces of analytic in the unit disk $\mathbb{D}$ functions: in particular, $H^{\infty}$ is the space of all bounded analytic in $\mathbb{D}$ functions;

$H_{E}^{2} \quad$ vector-valued Hardy class $H^{2}$ with values in $E$; if $\operatorname{dim} E=n$ we often identify $E$ with $\mathbb{C}^{n}$ and use the notation $H_{n}^{2}$.

$L_{E \rightarrow E_{*}}^{\infty} \quad$ class of bounded functions on the unit circle $\mathbb{T}$ whose values are bounded operators from $E$ to $E_{*}$;

$H_{E \rightarrow E_{*}}^{\infty} \quad$ operator Hardy class of bounded analytic functions whose values are bounded operators from $E$ to $E_{*}$;

$$
\|F\|_{\infty}:=\sup _{z \in \mathbb{D}}\|F(z)\|=\operatorname{esssup}_{\xi \in \mathbb{T}}\|F(\xi)\| .
$$

If $\operatorname{dim} E=n<\infty, \operatorname{dim} E_{*}=m<\infty$,we often identify $E$ with $\mathbb{C}^{n}$ and $E_{*}$ with $\mathbb{C}^{m}$ and use symbol $H_{m \times n}^{\infty}$.

$H_{\Phi}, T_{\Phi} \quad$ Hankel and Toeplitz operators with symbol $\Phi$.

Throughout the paper all Hilbert spaces are assumed to be separable. We always assume that in any Hilbert space an orthonormal basis is fixed, so any operator $A: E \rightarrow E_{*}$ can be identified with its matrix. Thus besides usual involution $A \mapsto A^{*}$ ( $A^{*}$ is the adjoint of $A$ ), we will have two more: $A \mapsto A^{T}$ (transpose of the matrix) and $A \mapsto \bar{A}$ (complex conjugation of the matrix), so $A^{*}=(\bar{A})^{T}=\overline{A^{T}}$. Although everything in the paper can be presented in invariant, "coordinate-free" form, use of transposition and complex conjugation makes the notation easier and more transparent.

\section{INTRODUCTION}

0.1. What is the Operator (Matrix) Corona Problem? The Operator Corona Problem is the problem of finding a (preferably local) necessary and sufficient condition for a bounded operator-valued function $F \in H_{E \rightarrow E_{*}}^{\infty}$ to have a left inverse in $H^{\infty}$, i.e. a function $G \in H_{E_{*} \rightarrow E}^{\infty}$ such that

$$
G(z) F(z) \equiv I \quad \forall z \in \mathbb{D} .
$$

If $\operatorname{dim} E<\infty$ we call it the Matrix Corona Problem.

The equations of type (B) are sometimes called in the literature the Bezout equations, and " $\mathrm{B}$ " here is for Bezout. The simplest necessary condition for $(\mathrm{B})$ is

$$
F^{*}(z) F(z) \geq \delta^{2} I, \quad \forall z \in \mathbb{D} \quad(\delta>0)
$$

(the tag "C" is for Carleson).

If the condition (C) implies (B), we say that the Operator (Matrix) Corona Theorem holds. 
The Operator Corona Theorem plays an important role in different areas of analysis, in particular in Operator Theory (angles between invariant subspaces, unconditionally convergent spectral decompositions, see $[6,7,14$, 15]), as well as in Control Theory and other applications.

Let us discuss the cases when the Operator Corona Theorem holds.

The first case is $\operatorname{dim} E=1, \operatorname{dim} E_{*}=n<\infty$. In this case $F=$ $\left[f_{1}, f_{2}, \ldots, f_{n}\right]^{T}, G=\left[g_{1}, g_{2}, \ldots, g_{n}\right]$ and it is simply the famous Carleson Corona Theorem [2], see also [4, Chapter VIII], [7, Appendix 3].

Later, using the ideas from the T. Wolff's proof of the Carleson Corona Theorem, M. Rosenblum [9], V. Tolokonnikov [12] and Uchiyama [17] independently proved that the Operator Corona Theorem holds if $\operatorname{dim} E=1$, $\operatorname{dim} E_{*}=\infty$.

Then, using simple linear algebra argument, P. Fuhrman [3] and V. Vasyunin $[12]^{1}$ independently proved that the Operator Corona Theorem holds if $\operatorname{dim} E<\infty, \operatorname{dim} E_{*} \leq \infty^{2}$. This theorem is now commonly referred to as the Matrix Corona Theorem.

And finally, a trivial observation: if $F(z) E=E_{*} \forall z \in \mathbb{D}$, then the left invertibility $(\mathrm{C})$ implies the invertibility of $F(z)$, and so we can simply put $G=F^{-1}$. So in this case the Operator Corona Theorem holds as well.

As for the general Operator Corona Theorem, it was shown by the author ([13], see also [14]) that it fails in the general case $\operatorname{dim} E=+\infty$

0.2. What is this paper about. This paper is devoted to the following two topics: codimension one conjecture and the estimates in the matrix $(\operatorname{dim} E<\infty)$ case.

0.2.1. Codimension one conjecture. Let us discuss the codimension one conjecture first. As we just mentioned above, the Operator Corona Theorem fails if $\operatorname{dim} E=\infty$, but it holds if $F(z) E=E_{*} \forall z \in \mathbb{D}$. So, what happens if the operators $F(z)$ are "almost" onto, namely if $\operatorname{codim}(F(z) E)=1$ $\forall z \in \mathbb{D}$ ? It was conjectured by N. Nikolski and the author that in this case the Operator Corona Theorem holds. It was also conjectured that the Matrix Corona Theorem holds for all $(n+1) \times n$ matrix-valued functions in $H_{(n+1) \times n}^{\infty}$ with uniform (independent on $n$ ) estimates. Of course, the Operator Corona Theorem holds in the matrix case by Fuhrmann-Vasyunin, the question is only in uniform estimates.

Besides the naïve reason that the codimension one case is very close to the case of invertible operator-function, there were some more deep facts that lead to the codimension one conjecture. Namely, see Lemma 2.3 and Theorem 2.4 below, with each $(n+1) \times n$ matrix function in $H^{\infty}$ one can canonically associate an $(n+1) \times 1$ matrix (vector) with the same best norm of a left inverse.

\footnotetext{
${ }^{1}$ It is not a typo, Vasyunin's result was indeed published (with attribution) in the Tolokonnikov's paper.

${ }^{2}$ To be precise, P. Fuhrman treated only the case $\operatorname{dim} E_{*}<\infty$
} 
Since in $n \times 1$ case it is possible to obtain the estimate in the Corona Theorem independent of $n$, it seemed reasonable to propose the codimension one conjecture, at least in its matrix version.

One of the main results of this paper is that the codimension one conjecture is false. It is interesting that the above canonically associated vector (the so called co-analytic complement) plays an important role in the construction.

Other inportant results in are Theorem 2.4 and Corollary 2.6 which clarify the role of co-analytic orthogonal complements in the Operator Corona Problem.

0.2.2. Lower bounds in the Matrix Corona Theorem. The second problem we are dealing with in this paper is the problem of estimates in the matrix case. It was shown by V. Vasyunin [12] (see also [16]) that if $\operatorname{dim} E=n<\infty$ and we normalize the function $F \in H_{E \rightarrow E_{*}}^{\infty}$ as

$$
I \geq F^{*}(z) F(z) \geq \delta^{2} I, \quad \forall z \in \mathbb{D},
$$

then one can always find a left inverse $G \in H_{E_{*} \rightarrow E}^{\infty}, G F \equiv I$, such that $\|G\|_{\infty} \leq C(n, \delta)$, where

$$
C(n, \delta)=C \sqrt{n} \cdot \delta^{-2 n} \log \delta^{-2 n} .
$$

Recently, T. Trent [16] was able to improve this estimate with

$$
C(n, \delta)=C \cdot \delta^{-n-1} \log \delta^{-2 n},
$$

but in both cases $C(n, \delta)$ grows exponentially in $n$.

On the other hand, the author had shown in [13], see also [14, Chapter $5]$ that $C(n, \delta)$ cannot be uniformly (in $n$ ) bounded, namely that for any sufficiently small $\delta>0$ one can find $F \in H_{E \rightarrow E_{*}}^{\infty}, \operatorname{dim} E=n$, satisfying (0.1) and such that any left inverse $G \in H_{E_{*} \rightarrow E}^{\infty}$ satisfies the inequality

$$
\|G\|_{\infty} \geq c(n, \delta)=C \delta^{-2} \log \left(\delta^{2} n+1\right)
$$

so $c(n, \delta)$ grows logarithmically in $n$. From this estimates it is easy to get that the operator Corona Theorem fails in the general case $\operatorname{dim} E=\infty$.

In this paper we prove the lower bound $[\delta /(1-\delta)]^{-n}$ which is quite close to Trent's estimate. so his estimate is probably very close to a sharp one. Moreover, this estimate is obtained for $(n+1) \times n$ matrices, so it disproves the codimension one conjecture (its matrix version).

For the exact statements the reader is referred to the next section.

\section{MAIN RESUlts}

Theorem 1.1. Given $\delta, 0<\delta<1 / 3$ and $n \in \mathbb{N}$ there exists an $(n+1) \times n$ inner matrix function $F \in H_{(n+1) \times n}^{\infty}$ such that

$$
I \geq F^{*}(z) F(z) \geq \delta^{2} I \quad \forall z \in \mathbb{D},
$$

but the norm of any left inverse for $F$ is at least $[\delta /(1-\delta)]^{-n} \geq\left(\frac{3}{2} \delta\right)^{-n}$. 
Theorem 1.2. Given $\delta, 0<\delta<1 / 3$ there exists an inner operator valued function $F \in H_{E \rightarrow E_{*}}^{\infty}(\operatorname{dim} E=\infty)$ such that

$$
I \geq F^{*}(z) F(z) \geq \delta^{2} I \quad \forall z \in \mathbb{D},
$$

the codimension of $F(z) E$ is $E_{*}$ is one $\forall z \in \mathbb{D}$, but $F$ does not have a left inverse in $H^{\infty}$. Moreover, the function $F$ can be chosen such that $\operatorname{codim}(F(z) E)=1$ a.e. on $\mathbb{T}$, or such that $F(z)$ is onto a.e. on $\mathbb{T}$.

\section{Co-Analytic complements and their role in the Corona} PROBLEM

Recall that a function $F \in H_{E \rightarrow E_{*}}^{\infty}$ is called inner if operators $F(z)$ are isometries a.e. on $\mathbb{T}$, and an outer if $F H_{E}^{2}$ is dense in $H_{E_{*}}^{2}$. We will call $F$ co-inner (resp. co-outer) if $F^{T}$ is inner (resp. outer).

Let us recall that any $F \in H_{E \rightarrow E_{*}}^{\infty}$ admits an inner-outer factorization $F=F_{\mathrm{i}} F_{\mathrm{o}}$, where $F_{\mathrm{i}} \in H_{E_{1} \rightarrow E_{*}}^{\infty}$ is inner and $F_{\mathrm{o}} \in H_{E \rightarrow E_{1}}^{\infty}$ is outer. Let us recall also that the inner part $F_{\mathrm{i}}$ (resp. the outer part $F_{0}$ ) is unique up to a constant unitary factor on the right (resp. on the left).

Let us also recall that any $z$-invariant subspace $M(z M \subset M)$ of $H_{E}^{2}$ can be represented as $\Theta H_{E_{1}}^{2}$, where $E_{1}$ is an auxiliary Hilbert space and $\Theta \in H_{E_{1} \rightarrow E}^{\infty}$ is an inner function.

\subsection{Co-analytic orthogonal complements.}

Definition 2.1. We say that an operator valued function $v \in H_{E \rightarrow E_{*}}^{\infty}$ has a bounded co-analytic (orthogonal) complement if there exists a function $V \in H_{E_{1} \rightarrow E_{*}}^{\infty}$ such that $\operatorname{ker} v^{T}(z)=V(z) E_{1}$ a.e. on $\mathbb{T}$. The function $\bar{V}$ is called a co-analytic (orthogonal) complement of $v$.

We will usually in this paper skip the word orthogonal, and simply say co-analytic complement.

The reason for the word co-analytic complement is the that the equality $\operatorname{ker} v^{T}(z)=V(z) E_{1}$ can be rewritten as

$$
\bar{V}(z) E_{1}=(v(z) E)^{\perp} \quad \text { a.e. on } \mathbb{T} .
$$

Without loss of generality (take the inner part of $V$ ) we can always assume that the function $V$ is inner. Moreover, by taking the outer part of $V^{T}$ we can always assume that $V$ is also co-outer. So, when we say the co-analytic complement we usually mean $\bar{V}$, where $V$ is an inner and co-outer function. Lemma 2.2 below states that such inner and co-outer function is unique up to a constant unitary factor on the right.

Let us also make a simple observation, that if the function $v$ is inner (i.e. operators $v(z)$ are isometries a.e. on $\mathbb{T}$ ), then the operators $W(z)$, where $W=[v \bar{V}] \in H_{E \oplus E_{1} \rightarrow E_{*}}^{\infty}$ is defined by

$$
W(z)\left(\boldsymbol{e} \oplus \boldsymbol{e}_{1}\right)=v(z) \boldsymbol{e}+\bar{V}(z) \boldsymbol{e}_{1}, \quad \boldsymbol{e} \in E, \boldsymbol{e}_{1} \in E_{1}
$$

are unitary a.e. on $\mathbb{T}$. 
Lemma 2.2. Let $v \in H_{E \rightarrow E_{*}}^{\infty}$ have a bounded co-analytic complement $\bar{V}$, where $V \in H_{E_{1} \rightarrow E_{*}}^{\infty}$ is an inner and co-outer function such that

$$
\operatorname{ker} v^{T}(z)=V(z) E_{1} \quad \text { a.e. on } \mathbb{T} .
$$

Then

$$
\left\{f \in H_{E_{*}}^{2}: v^{T} f \equiv 0 \text { a.e. on } \mathbb{T}\right\}=V H_{E_{1}}^{2} \text {. }
$$

Moreover, any other such inner and co-outer function $V_{2} \in H_{E_{2} \rightarrow E_{*}}^{\infty}$ satisfies $V_{2}=V U$, where $U: E_{2} \rightarrow E_{1}$ is a constant unitary operator.

Proof. Denote

$$
M:=\left\{f \in H_{E_{*}}^{2}: v^{T} f \equiv 0 \text { a.e. on } \mathbb{T}\right\} .
$$

Clearly, $V H_{E_{1}}^{2} \subset M$. Since $z M \subset M$,

$$
M=\widetilde{V} H_{\widetilde{E}}^{2},
$$

where $\widetilde{V} \in H_{\widetilde{E} \rightarrow E_{*}}^{\infty}$ is an inner function.

Since $V H_{E_{1}}^{2} \subset M=\widetilde{V} H_{\widetilde{E}}^{2}$, the preimage $\widetilde{V}^{-1}\left(V H_{E_{1}}^{2}\right)$ is a $z$-invariant subspace of $H_{\widetilde{E}}^{2}$. So it can be represented as $\widetilde{V}^{-1}\left(V H_{E_{1}}^{2}\right)=U H_{E_{U}}^{2}$, where $U \in H_{E_{U} \rightarrow \widetilde{E}}^{\infty}$ is an inner function and $E_{U}$ is an auxiliary Hilbert space. Therefore $V H_{E_{1}}^{2}=\widetilde{V} U H_{E_{U}}^{2}$, so the space $E_{U}$ can be identified with $E_{1}$ and the function $V$ can be factorized as $V=\widetilde{V} U$. Therefore $V(z) E_{1} \subset \widetilde{V}(z) E_{2}$ a.e. on $\mathbb{T}$, and so

$$
\operatorname{ker} v(z)^{T}=V(z) E_{1} \subset \widetilde{V}(z) \widetilde{E} \subset \operatorname{ker} v(z)^{T} \quad \text { a.e. on } \mathbb{T} \text {. }
$$

This implies that $V(z) E_{1}=\widetilde{V}(z) \widetilde{E}$ and hence $U(z) E_{1}=\widetilde{E}$ a.e. on $\mathbb{T}$. This means that the function $U$ takes unitary values a.e. on $\mathbb{T}$, so $U^{T}$ is also an inner function. Since $V$ is a co-outer function, $U$ must be a constant (unitary operator).

The following well-known lemma asserts that a matrix valued function always has a bounded co-analytic complement.

Let $F \in H_{m \times n}^{\infty}$ be a matrix valued function. Since any minor of $F$ belongs to $H^{\infty}$, if it is non-zero on a set of positive measure in $\mathbb{T}$, then it is non-zero a.e. on $\mathbb{T}$. Recalling that the rank of a matrix $A$ is a maximal $k$ such that there exists a non-zero minor of order $k$, we can conclude rank $F(z) \equiv$ Const a.e. on $\mathbb{T}$. We will call this constant the rank of $F$ (and denote $\operatorname{rank} F$ ).

Lemma 2.3. Let $v$ be an $n \times m(m<n)$ matrix-valued function in $H^{\infty}=$ $H_{n \times m}^{\infty}$, and let rank $v=n-r$ a.e. on $\mathbb{T}$.

Then $v$ has a bounded co-analytic complement $\bar{V}$, where $V \in H_{n \times r}^{\infty}$ is an inner and co-outer function.

Proof. Consider the equation $v^{T} f \equiv 0$. Standard linear algebra argument implies that there exist vector functions $f_{1}, f_{2}, \ldots, f_{r}$ with entries in the 
meromorphic Nevanlina class $\mathcal{N}:=\left\{f / g: f, g \in H^{\infty}\right\}$ such that for almost all $z \in \mathbb{T}$

$$
\operatorname{ker} v^{T}(z)=\mathcal{L}\left\{f_{1}(z), f_{2}(z), \ldots, f_{r}(z)\right\}
$$

Multiplying all entries by the product of all denominators, we obtain that all vector functions $f_{1}, f_{2}, \ldots, f_{r}$ can be chosen to have entries in $H^{\infty}$.

Consider subspace $M \subset H_{n}^{2}$ consisting of all vectors $f \in H_{n}^{2}$ satisfying $v^{T} f \equiv 0$. Clearly $z M \subset M$, so $M$ can be represented as $M=V H_{r^{\prime}}^{2}$, where $V$ is an $n \times r^{\prime}$ inner function. Clearly, $r^{\prime} \leq r$ and $V(z) \mathbb{C}^{r^{\prime}} \subset \operatorname{ker} v(z)^{T}$ a.e. on $\mathbb{T}$.

Since $f_{1}, f_{2}, \ldots, f_{r} \in M=V H_{r}^{2}$, we have the opposite inclusion

$$
\operatorname{ker} v^{T}(z) \subset V(z) \mathbb{C}^{r^{\prime}} \quad \text { a.e. on } \mathbb{T} \text {. }
$$

Both inclusions together imply that $r^{\prime}=r$ and

$$
\operatorname{ker} v^{T}(z)=V(z) \mathbb{C}^{r} \quad \text { a.e. on } \mathbb{T} .
$$

The rest follows from Lemma 2.2.

2.2. Left invertibility and co-analytic complements. The theorem below shows relation between co-analytic complements and Corona Problem. Let us mention, that to prove Theorems 1.1 and 1.2 one can use a weaker version of it, namely the fact that the best possible norms of the left inverses of $F$ and $V$ coincide (provided that a co-analytic complement exists). Note, that such simple version was first proved by V. Peller and the author in [8]. However, it is always nice to have a complete understanding, so we present the theorem in full generality.

Theorem 2.4. Let $F \in H_{E \rightarrow E_{*}}^{\infty}$ be an inner function. Assume that $F$ is left invertible, but not right invertible in $H^{\infty}$. Then $F$ has an co-analytic complement $\bar{V}$, where $V \in H_{E_{1} \rightarrow E_{*}}^{\infty}$ is an inner and co-outer function. Moreover

$$
V(z) E_{1}=\operatorname{ker} F^{T}(z) \quad \forall z \in \mathbb{D},
$$

the function $V$ is also left invertible, and the best possible norms of left inverses for $F$ and $V$ coincide.

Remark 2.5. Note, that in the matrix case $\left(\operatorname{dim} E, \operatorname{dim} E_{*}<\infty\right)$ a simple dimension/rank counting shows that the equality

$$
V(z) E_{1}=\operatorname{ker} F^{T}(z) \quad \forall z \in \mathbb{D},
$$

and the same equality a.e. on $\mathbb{T}$ are equivalent.

In the general operator valued case it is not known whether one implies the other. Numerous counterexamples in the theory of analytic range functions, see [5] lead one to suspect that none of the implications holds.

The following corollary gives necessary and sufficient condition for the Operator Corona Theorem to be true in the case of finite codimension. 
Corollary 2.6. Let $F \in H_{E \rightarrow E_{*}}^{\infty}$ be an operator-valued function satisfying

$$
F(z)^{*} F(z) \geq \delta^{2} I \quad \forall z \in \mathbb{D},
$$

for some $\delta>0$. Assume that at a point $z \in \mathbb{D}$ (or on a set of positive measure in $\mathbb{T}) \operatorname{codim}(F(z) E)=n<\infty, n \neq 0$. Then the following statements are equivalent:

1. $F$ is left invertible in $H^{\infty}$, i.e. there exists $G \in H_{E_{*} \rightarrow E}^{\infty}$ such that $G F \equiv I$

2. There exists function $V \in H_{\mathbb{C}^{n} \rightarrow E_{*}}^{\infty}$ satisfying $V(z)^{*} V(z) \geq \widetilde{\delta}^{2} I \forall z \in \mathbb{D}$, $\widetilde{\delta}>0$, such that

$$
\left.V(z) \mathbb{C}^{n}=\operatorname{ker} F^{T}(z) \quad \forall z \in \mathbb{D} \quad \text { (and a.e. on } \mathbb{T}\right) .
$$

Moreover, the function $V$ always can be chosen to be inner, and if both $F$ and $V$ are inner, the best norms of the left inverses for $F$ and $V$ coincide.

Proof. Consider the inner-outer factorization of $F, F=F_{\mathrm{i}} F_{\mathrm{o}}$. Note, that

$$
F_{\mathrm{i}}^{*}(z) F_{\mathrm{i}}(z) \geq \widetilde{\delta}^{2} I, \quad \forall z \in \mathbb{D},
$$

where $\widetilde{\delta}=\delta /\|F\|_{\infty}$. The condition about the codimension of $F(z) E$ implies that $F$ (and therefore $F_{\mathrm{i}}$ ) is not invertible in $H^{\infty}$. Therefore, by Theorem 2.4 we have $1 \Longrightarrow 2$.

Since the Operator Corona theorem holds for functions in $H_{\mathbb{C}^{n} \rightarrow E_{*}}^{\infty}$, condition $V(z)^{*} V(z) \geq \widetilde{\delta}^{2} I$ implies that $V$ is left invertible. Since $\bar{V}_{\text {i }}$ is a co-analytic complement of $V$, Theorem 2.4 implies that $2 \Longrightarrow 1$.

To prove Theorem 2.4 we will need the following two well known results. Recall, that given $\Phi \in L_{E \rightarrow E_{*}}^{\infty}$, Hankel and Toeplitz operators $H_{\Phi}$ and $T_{\Phi}$ with symbol $\Phi$ are defined as

$$
\begin{array}{ll}
H_{\Phi}: H_{E}^{2} \rightarrow\left(H_{E_{*}}^{2}\right)^{\perp} & H_{\Phi} f:=P_{-}(\Phi f)^{\prime} \\
T_{\Phi}: H_{E}^{2} \rightarrow H_{E_{*}}^{2} & T_{\Phi} f:=P_{+}(\Phi f),
\end{array}
$$

where $P_{+}$and $P_{-}$are orthogonal projections onto $H^{2}$ and $\left(H^{2}\right)^{\perp}$ respectively.

Theorem 2.7 (Arveson [1], Sz.-Nagy-Foias [11]). Let $F \in H_{E \rightarrow E_{*}}^{\infty}$. The following two statements are equivalent:

1. The function $F$ is left invertible in $H^{\infty}$, i.e. there exists $G \in H_{E_{*} \rightarrow E}^{\infty}$ such that $G F \equiv I$;

2. The Toeplitz operator $T_{\bar{F}}$ is left invertible, that is

$$
\inf _{f \in H_{E}^{2},\|f\|=1}\left\|T_{\bar{F}} f\right\|=: \delta>0 .
$$

Moreover, the best possible norm of a left inverse $G$ is exactly $1 / \delta$.

This theorem also can be found in the monograph [6], see Theorem 9.2.1 there. 
Note, that this theorem is stated slightly differently in different papers. For example, Theorem 9.2.1 in [6] states that $F$ is right invertible in $H^{\infty}$ if and only if $T_{F^{*}}$ is left invertible: applying it to $F^{T}$ we get the statement of Theorem 2.7. Similarly, the theorem in [11] states that $F$ is left invertible in $H^{\infty}$ if and only if $T_{F^{\#}}$ is left invertible, where $F^{\#}(z):=F(\bar{z})$. Again, applying this theorem to $\overline{F(\bar{z})}$ we get Theorem 2.7 .

Lemma 2.8. Let $F \in L_{E \rightarrow E_{*}}^{\infty}$, and let $F(\xi)$ be an isometry a.e. on $\mathbb{T}$. Then Toeplitz operator $T_{F}$ is left invertible if and only if $\left\|H_{F}\right\|<1\left(H_{F}\right.$ is the Hankel operator). Moreover, $\left\|H_{F}\right\|^{2}=1-\delta^{2}$, where

$$
\delta:=\inf _{f \in H_{E}^{2},\|f\|=1}\left\|T_{F} f\right\|
$$

Proof. Clearly $F f=T_{F} f+H_{F} f$ for $f \in H_{E}^{2}$, so

$$
\|f\|^{2}=\|F f\|^{2}=\left\|T_{F} f\right\|^{2}+\left\|H_{F} f\right\|^{2},
$$

and the lemma follows immediately.

Proof of Theorem 2.4. Let $G \in H_{E_{*} \rightarrow E}^{\infty}$ be a left inverse of $F$, that is $G F \equiv$ I. Then $F^{T} G^{T} \equiv I$, and for $\mathcal{P} \in H_{E_{*} \rightarrow E_{*}}^{\infty}, \mathcal{P}:=G^{T} F^{T}$ we have

$$
\mathcal{P}^{2}=G^{T}\left(F^{T} G^{T}\right) F^{T}=G^{T} F^{T}=\mathcal{P},
$$

so $\mathcal{P}(z)$ is a projection a.e. on $\mathbb{T}$ (and $\forall z \in \mathbb{D}$ ). Since

$$
\mathcal{P}(z) G^{T}(z) \boldsymbol{e}=G(z)^{T} F(z)^{T} G(z)^{T} \boldsymbol{e}=G(z)^{T} \boldsymbol{e} \quad \forall \boldsymbol{e} \in E_{*},
$$

we can conclude that $\mathcal{P}(z) E_{*}=G(z)^{T} E$ a.e. on $\mathbb{T}$, as well as $\forall z \in \mathbb{D}$. Since $G^{T}$ is left invertible, $\operatorname{ker} \mathcal{P}(z)=\operatorname{ker} F(z)^{T}$ a.e. on $\mathbb{T}$ (and $\forall z \in \mathbb{D}$ ). So, for the complementary projection $\mathcal{Q}(z), \mathcal{Q}:=I-\mathcal{P}$ we have

$$
\mathcal{Q}(z) E_{*}=\operatorname{ker} F(z)^{T}, \quad \text { a.e. on } \mathbb{T} \quad(\text { and } \forall z \in \mathbb{D}) \text {. }
$$

Note, that $\mathcal{Q}(z) \not \equiv 0$ because $F$ is not right invertible in $H^{\infty}$. Taking $V$ to be the inner part of $\mathcal{Q}$ we see that $F$ has a co-analytic complement.

Now let us prove the rest of the theorem. Since $F$ is left invertible, Theorem 2.7 implies that

$$
\inf _{f \in H_{E}^{2},\|f\|=1}\left\|T_{\bar{F}} f\right\|=: \delta>0 .
$$

By Lemma $2.8\left\|H_{\bar{F}}\right\|^{2}=1-\delta^{2}$ (recall that $F(\xi)$ is an isometry a.e. on $\mathbb{T}$ ).

Consider the operator valued function $W=[\bar{F}, V] \in H_{E \oplus E_{1} \rightarrow E_{*}}^{\infty}$,

$$
W(z)\left(\boldsymbol{e} \oplus \boldsymbol{e}_{1}\right)=\bar{F}(z) \boldsymbol{e}+V(z) \boldsymbol{e}_{1}, \quad \boldsymbol{e} \in E, \boldsymbol{e}_{1} \in E_{1}, \quad z \in \mathbb{T},
$$

where $V \in H_{E_{1} \rightarrow E_{*}}^{\infty}$ is the inner and co-outer function from the definition of the co-analytic complement. Recall (see the beginning of the section) that the function $W$ takes unitary values a.e. on $\mathbb{T}$.

Consider Hankel operator $H_{W}$. Its symbol is obtained from $\bar{F}$ by adding an analytic block, so $H_{W}$ differs from $H_{\bar{F}}$ by a zero block. Hence

$$
\left\|H_{W}\right\|^{2}=\left\|H_{\bar{F}}\right\|^{2}=1-\delta^{2}
$$


and the Toeplitz operator $T_{W}$ is left invertible.

Let us show that the adjoint operator $T_{W}^{*}=T_{W^{*}}$ has trivial kernel. Indeed, let $T_{W^{*}} f=0, f \in H_{E_{*}}^{2}$. Then

$$
W^{*} f=\left[\begin{array}{c}
F^{T} f \\
V^{*} f
\end{array}\right] \in\left(H_{E \oplus E_{1}}^{2}\right)^{\perp} .
$$

Since $F \in H_{E \rightarrow E_{*}}^{\infty}$, this implies $F^{T} f \equiv 0$. By Lemma $2.2 f=V g, g \in H_{E}^{2}$, thus $g \equiv 0$ and therefore $f \equiv 0$. So $\operatorname{ker} T_{W^{*}}=\{0\}$.

The operator $T_{W}$ is left invertible and $\operatorname{ker} T_{W}^{*}=\{0\}$, that means $T_{W}$ is invertible. By Lemma $2.8\left\|H_{W^{*}}\right\|^{2}=1-\delta^{2}$. Since $W^{*}$ differs from $V^{*}$ by an analytic block, Hankel operators $H_{V^{*}}$ and $H_{W^{*}}$ differ by a zero block, thus

$$
\left\|H_{V^{*}}\right\|^{2}=\left\|H_{W^{*}}\right\|^{2}=\left\|H_{W^{*}}\right\|^{2}=1-\delta^{2} .
$$

By the Nehari theorem $\left\|H_{V^{*}}\right\|=$ dist $_{L^{\infty}}\left(V^{*}, H^{\infty}\right)$, and since the transposition does not change the norm in an operator valued $L^{\infty}$ and $\bar{V}=\left(V^{*}\right)^{T}$,

$$
\left\|H_{\bar{V}}\right\|=\operatorname{dist}_{L^{\infty}}\left(\bar{V}, H^{\infty}\right)=\operatorname{dist}_{L^{\infty}}\left(V^{*}, H^{\infty}\right)=\left\|H_{V^{*}}\right\|=\sqrt{1-\delta^{2}} .
$$

Therefore, by Theorem 2.7 the Toeplitz operator $T_{\bar{V}}$ is left invertible, and thus $V$ is left invertible in $H^{\infty}$.

\section{Main construction and the proof of Theorem 1.1.}

For $\delta<1 / 3$ let $\alpha>0$ be a small number such that

$$
\frac{\delta}{1-\alpha}=: \delta^{\prime}<\frac{1}{3}
$$

Define $a:=\delta^{\prime} /\left(1-\delta^{\prime}\right)$, so $a /(1+a)=\delta^{\prime}$. Note, that $a<1 / 2$.

Consider $(n+1) \times n$ matrix $F \in H^{\infty}$,

$$
F=\left(\begin{array}{lllccc}
\varphi_{1}(z) & 0 & 0 & 0 & \ldots & 0 \\
-a & \varphi_{2}(z) & 0 & 0 & \ldots & 0 \\
0 & -a & \varphi_{3}(z) & 0 & \ldots & 0 \\
0 & 0 & -a & \varphi_{4}(z) & \ldots & 0 \\
\vdots & \vdots & \vdots & \ddots & \ddots & \vdots \\
0 & 0 & 0 & 0 & \ddots & \varphi_{n}(z) \\
0 & 0 & 0 & 0 & \ldots & -a
\end{array}\right)
$$

where $\varphi_{k}$ are some inner functions to be chosen later. Another way to describe $F$ is to say that its columns $F_{k}$ can be represented as

$$
F_{k}=\varphi_{k}(z) e_{k}-a e_{k+1}
$$

where $\boldsymbol{e}_{k}, k=1,2, \ldots, n+1$ is the standard basis in $\mathbb{C}^{n+1}$. 
It is easy to check that the $n+1$ dimensional vector-function (column)

$$
V=\left(\frac{1-a^{2}}{1-a^{2 n+2}}\right)^{1 / 2}\left(\begin{array}{c}
a^{n} \\
a^{n-1} \varphi_{1} \\
a^{n-2} \varphi_{1} \varphi_{2} \\
\ldots \\
a \varphi_{1} \varphi_{2} \ldots \varphi_{n-1} \\
\varphi_{1} \varphi_{2} \ldots \varphi_{n}
\end{array}\right)
$$

is inner and co-outer, and that $\bar{V}$ is orthogonal to the columns of $F$. Let us now assume that $\varphi_{n}$ has zero, for example that $\varphi_{n}(0)=0$. Then $\|V(0)\|=$ $a^{n} \sqrt{\left(1-a^{2}\right) /\left(1-a^{2 n+2}\right)}<a^{n} / \sqrt{1+a^{2}}$, so the norm of a left inverse to the column $V$ is at least $\sqrt{1+a^{2}} / a^{n}$.

It is easy to see that $\|F\|_{\infty} \leq 1+a$ (1 comes from the main diagonal, $a$ from the one below).

Suppose, that we can pick the inner functions $\varphi_{k}$ such that

$$
\|F(z) \boldsymbol{e}\| \geq(1-\alpha) a\|\boldsymbol{e}\|, \quad \forall z \in \mathbb{D}, \forall \boldsymbol{e} \in \mathbb{C}^{n}
$$

where $\alpha>0$ is from the beginning of this section.

Then for the inner part $F_{\mathrm{i}}$ of $F$

$$
\|\boldsymbol{e}\| \geq\left\|F_{\mathrm{i}}(z) \boldsymbol{e}\right\| \geq \frac{(1-\alpha) a}{1+a}\|\boldsymbol{e}\|=\delta\|\boldsymbol{e}\|, \quad \forall z \in \mathbb{D}, \forall \boldsymbol{e} \in \mathbb{C}^{n}
$$

Theorem 2.7 implies that the norm of an analytic left inverse to $F_{\mathrm{i}}$ is at least $\sqrt{1+a^{2}} / a^{n}$. Since

$$
\delta^{\prime}=\frac{\delta}{1-\alpha} \rightarrow \delta, \quad a=\frac{\delta^{\prime}}{1+\delta^{\prime}} \rightarrow \frac{\delta}{1+\delta} \quad \text { as } \alpha \rightarrow 0
$$

and $\alpha$ can be chosen arbitrarily small, we can construct a function $F_{\mathrm{i}}$ such that the norm of any $H^{\infty}$ left inverse is at least $[\delta /(1-\delta)]^{-n}$ and the theorem is proved.

To construct functions $\varphi_{k}$, pick an integer $N$ such that $(2 a)^{N}<\alpha a$. Put $\varphi_{1}(z)=z, \varphi_{2}(z)=z^{N}, \varphi_{3}(z)=z^{N^{2}}, \ldots, \varphi_{n}(z)=z^{N^{n-1}}$. In other words, $\varphi_{1}(z) \equiv z, \varphi_{n+1}=\varphi_{n}^{N}$. Then for any fixed $z$ the inequality

$$
\alpha a \leq \varphi_{k}(z) \leq 2 a
$$

holds for at most one $k=k(z)$ (it may happen that for a some $z$ it does not hold for any $k$ ). Fix $z \in \mathbb{D}$, and let $k=k(z)$ be such number. Then in the 
matrix $F(z)$ below

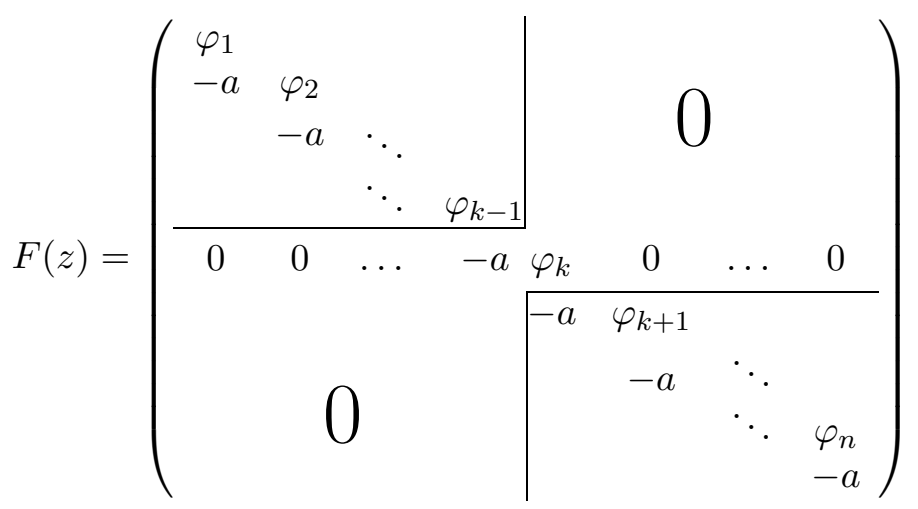

in both, upper left and lower right blocks, the main diagonal dominates the other non-trivial one. Therefore, both the upper left and the lower right blocks are invertible with the estimates of the norm of the inverse $1 / a$ and $a^{-1}(1-\alpha)^{-1}$ respectively. So, if we delete the $k$ th row, we get a (block diagonal) invertible operator with the norm of the inverse at most $a^{-1}(1-\alpha)^{-1}$.

Since adding an extra row does not spoil left invertibility, we conclude that $F(z)$ is left invertible and

$$
\|F(z) \boldsymbol{e}\| \geq(1-\alpha) a\|\boldsymbol{e}\|, \quad \forall \boldsymbol{e} \in \mathbb{C}^{n}
$$

\section{Proof of Theorem 1.2 .}

Consider an operator-valued function $F$,

$$
F=\left(\begin{array}{lllclcl}
\varphi_{1}(z) & 0 & 0 & 0 & \ldots & 0 & \ldots \\
-a & \varphi_{2}(z) & 0 & 0 & \ldots & 0 & \ldots \\
0 & -a & \varphi_{3}(z) & 0 & \ldots & 0 & \ldots \\
0 & 0 & -a & \varphi_{4}(z) & \ldots & 0 & \ldots \\
\vdots & \vdots & \vdots & \ddots & \ddots & \vdots & \\
0 & 0 & 0 & 0 & \ddots & \varphi_{n}(z) & \ldots \\
0 & 0 & 0 & 0 & \ldots & -a & \ldots \\
\vdots & \vdots & \vdots & \vdots & & \vdots & \ddots
\end{array}\right)
$$

which is an infinite dimensional analogue of the function $F$ constructed in the previous section. Here $a, \varphi_{1}, \ldots, \varphi_{n}, \ldots$ are exactly the same as in Section 3. As in Section 3 we can show that

$$
(1+a)^{2} I \geq F^{*}(z) F(z) \geq(1-\alpha)^{2} a^{2} I \geq \delta^{2} I .
$$


Note, that for $z \in \mathbb{D}$ operator $F(z)$ is not onto: subspace $(F(z) E)^{\perp}$ is spanned by the vector

$$
\bar{V}:=\left(\begin{array}{c}
1 \\
\bar{\varphi}_{1} / \bar{a} \\
\bar{\varphi}_{1} \bar{\varphi}_{2} / \bar{a}^{2} \\
\vdots \\
\bar{\varphi}_{1} \bar{\varphi}_{2} \ldots \bar{\varphi}_{n} / \bar{a}^{n} \\
\vdots
\end{array}\right)
$$

On the other hand, it is easy to see that the operators $F(z)$ are invertible a.e. on $\mathbb{T}$ (the main diagonal dominates), so according to Corollary $2.6, F$ does not have a left inverse. To get the statement about inner function, it is sufficient to take the inner part of $F$.

To prove that it is possible to pick $F$ such that $F(z) E$ has codimension 1 a.e. on $\mathbb{T}$, let us first notice that for the vector $V$ above

$$
\lim _{|z| \rightarrow 1-}\|V(z)\|=\infty \text {. }
$$

Therefore it is possible to find a simply connected domain $D \subset \mathbb{D}$ with $C^{\infty}$-smooth boundary, which touches $\mathbb{T}$ exactly at one point, such that

$$
\int_{\partial D} \log \|V(z)\| \cdot|d z|=+\infty
$$

Since for such domains the harmonic measue is equivalent to the arclength $|d z|$, we can replace $|d z|$ by the harmonic measure, and still get $+\infty$ in the integral. Note also, that everywhere on $\partial D$ except the point $\partial D \cap \mathbb{T}$, the codimension of $F(z) E$ is 1 .

So, if $\omega: \mathbb{D} \rightarrow D$ is a conformal mapping, then for $F_{1}:=F \circ \omega, V_{1}:=V \circ \omega$ we have that for all $z \in \operatorname{clos} \mathbb{D}$ except one point on $\mathbb{T}$

$$
\left(F_{1}(z) E\right)^{\perp}=\operatorname{span} \bar{V}_{1}(z) .
$$

Notice, that also

$$
\lim _{r \rightarrow 1-} \int_{r \mathbb{T}} \log \left\|V_{1}(z)\right\| \cdot|d z|=\int_{\mathbb{T}} \log \left\|V_{1}(z)\right\| \cdot|d z|=+\infty .
$$

But it is easy to see that $F_{1}$ is not left invertible in $H^{\infty}$. Indeed, if $F$ is left invertible in $H^{\infty}$, Corollary 2.6 asserts that there exist a vector function $v \in H_{E_{*}}^{\infty}=H_{\mathbb{C} \rightarrow E_{*}}^{\infty}$ such that

$$
(F(z) E)^{\perp}=\operatorname{span} \bar{v}(z)
$$

for all $z \in \mathbb{D}$ and a.e. on $\mathbb{T}$. Any such function must be represented as $v(z)=u(z) V_{1}(z)$ where $u$ is a scalar function. Since $\left\|V_{1}(z)\right\| \geq 1$ the function $u$ must be bounded, and since both $v$ and $V_{1}$ are holomorphic in $\mathbb{D}$, the function $u$ also must be holomorphic. Therefore $u \in H^{\infty}$, which is impossible because of (4.1). 


\section{SOME OPEN PROBlEMS.}

In this section we discuss some open problem concerning the Operator Corona Problem. Of course, the ultimate problem is to find a local necessary and sufficient condition for left invertibility of $F \in H_{E \rightarrow E_{*}}^{\infty}$. But this is probably hopeless, so there are several problems that seem to be more tractable.

5.1. Operator Corona for large $\delta$. Does there exist $\delta>0$ (close to 1 ) such that for any $F \in H_{E \rightarrow E_{*}}^{\infty}$ the inequality

$$
I \geq F(z)^{*} F(z) \geq \delta^{2} I
$$

implies that there exists $G \in H_{E_{*} \rightarrow E}^{\infty}$ such that $G F \equiv I$ ?

The counterexample constructed in this paper works only for $\delta<1 / 3$, the method from [13] gives counterexample for $\delta<1 / \sqrt{2}$.

5.2. Role of the co-analytic complement. As Theorem 2.4 asserts, if $F \in H_{E \rightarrow E_{*}}^{\infty}$ is invertible, there exists a left invertible $G \in H_{E_{1} \rightarrow E_{*}}^{\infty}$ such that ker $F(z)^{T}=V(z) E_{1}$ a.e. on $\mathbb{T}$.

Suppose $F \in H_{E \rightarrow E_{*}}^{\infty}$ satisfies

$$
F(z)^{*} F(z) \geq \delta^{2} I \quad \forall z \in \mathbb{D},
$$

and suppose we know that there exists $V H_{E_{1} \rightarrow E_{*}}^{\infty}$ such that $\operatorname{ker} F(z)^{T}=$ $V(z) E_{1}$ a.e. on $\mathbb{T}$ also satisfying the Corona Condition

$$
V(z)^{*} V(z) \geq \varepsilon^{2} I
$$

Does this imply that $F$ is left invertible in $H^{\infty}$ ?

\section{REFERENCES}

[1] W. Arveson, Interpolation problems in nest algebras, J. Functional Analysis 20 (1975), no. 3, 208-233.

[2] L. Carleson, Interpolations by bounded analytic functions and the Corona problem, Ann. of Math. (2) $\mathbf{7 6}$ (1962), 547-559.

[3] P. Fuhrmann, On the corona theorem and its application to spectral problems in Hilbert space, Trans. Amer. Math. Soc. 132 (1968), 55-66.

[4] J. B. Garnett, Bounded analytic functions, Pure and Applied Mathematics, vol. 96, Academic Press Inc. [Harcourt Brace Jovanovich Publishers], New York, 1981.

[5] H. Helson, Lectures on invariant subspaces, Academic Press, New York, 1964.

[6] N. K. Nikolski, Operators, functions, and systems: an easy reading. Vol. 1: Hardy, Hankel, and Toeplitz, Mathematical Surveys and Monographs, vol. 92, American Mathematical Society, Providence, RI, 2002, Translated from the French by Andreas Hartmann.

[7] - Treatise on the shift operator, Grundlehren der Mathematischen Wissenschaften [Fundamental Principles of Mathematical Sciences], vol. 273, SpringerVerlag, Berlin, 1986, Spectral function theory, With an appendix by S. V. Hruščev [S. V. Khrushchëv] and V. V. Peller, Translated from the Russian by Jaak Peetre.

[8] V. V. Peller and S. R. Treil, Approximation by analytic matrix functions: the four block problem, J. Funct. Anal. 148 (1997), no. 1, 191-228.

[9] M. Rosenblum, A corona theorem for countably many functions, Integral Equations Operator Theory 3 (1980), no. 1, 125-137. 
[10] B. Sz.-Nagy and C. Foiaş, Harmonic analysis of operators on Hilbert space, Translated from the French and revised, North-Holland Publishing Co., Amsterdam, 1970.

[11] On contractions similar to isometries and Toeplitz operators, Ann. Acad. Sci. Fenn. Ser. A I Math. 2 (1976), 553-564.

[12] V. A. Tolokonnikov, Estimates in the Carleson corona theorem, ideals of the algebra $H^{\infty}$, a problem of Sz.-Nagy, Zap. Nauchn. Sem. Leningrad. Otdel. Mat. Inst. Steklov. (LOMI) 113 (1981), 178-198, 267, Investigations on linear operators and the theory of functions, XI.

[13] S. R. Treil, Angles between co-invariant subspaces, and the operator corona problem. The Szökefalvi-Nagy problem, Dokl. Akad. Nauk SSSR 302 (1988), no. 5, 1063-1068.

[14] _ Geometric methods in spectral theory of vector-valued functions: some recent results, Toeplitz operators and spectral function theory, Oper. Theory Adv. Appl., vol. 42, Birkhäuser, Basel, 1989, pp. 209-280.

[15] _ Unconditional bases of invariant subspaces of a contraction with finite defects, Indiana Univ. Math. J. 46 (1997), no. 4, 1021-1054.

[16] T. Trent, A new estimate for the vector valued corona problem, J. Funct. Anal. 189 (2002), no. 1, 267-282.

[17] A. Uchiyama, Corona theorems for countably many functions and estimates for their solutions, preprint, UCLA, 1980.

Department of Mathematics, Brown University, 151 Thayer Str., Box 1917, Providence, RI 02912, USA

E-mail address: treil@math.brown.edu

URL: http://www. math. brown.edu/ ${ }^{\sim}$ treil 